Mental health and the criminal justice system: the role of interagency training to promote practitioner understanding of the diversion agenda

\title{
Mental health and the criminal justice system: the role of interagency training to promote practitioner understanding of the diversion agenda
}

\begin{abstract}
Historically there has been a significant under-recognition of mental health problems amongst people in the criminal justice system, and little research exploring the issues encountered by those with mental health problems who come into contact with the criminal justice system. Recent policy has highlighted the importance of early identification of mental health needs in criminal cases, and the role of diversion of offenders into appropriate mental health services. However research suggests that currently the provision of mental health services for offenders is patchy, and it has been suggested that improved interagency communication and training is required to improve the diversion of offenders with mental health problems into more appropriate mental health provision.
\end{abstract}

The aim of this paper is to consider the current position of those with mental health conditions within the criminal justice system in England, and discuss how joint interagency training can improve understanding of the diversion agenda for the range of practitioners that come into contact with offenders with mental health problems. The perspectives of a range of practitioners who attended a joint interagency training day will be discussed, and recommendations for future training will be offered.

Key words: Mental health, criminal justice, diversion, and interagency training.

\section{Introduction}


Historically there has been a significant under-recognition of mental health problems amongst people in contact with the criminal justice system (CJS) and as a result the incidence of mental illness within prisons internationally is unacceptably high (Van Marle, 2007). This is a particular concern in the United Kingdom (Ministry of Justice, 2007; Department of Health, 2007a).

This issue is compounded by limited research specifically concerned with the experiences of court users with mental health conditions (McLeod et al, 2010). Prisoners are probably the most socially excluded group in our society (Rutherford et al., 2008), and studies report a high number of prisoners with mental health problems, with nearly all prisoners in England having one or more mental health problems (Singleton et al., 1998).

Some theorists have suggested that both prisons and mental health hospitals have been used as a method of punitive containment to 'invisibilize' marginal groups and problem populations (Wacquant, 2010:199). Harcourt (2006) identifies a history of incarceration within the USA which reached a peak in the 1950s, whereby such marginalized groups were either detained in prison or long stay mental institutions. The process of de-instituionalisation of people with long-term mental illnesses in recent years has shone a different lens on this issue, and the reduction in the number of psychiatric beds may contribute to increasing numbers of mentally-ill prisoners (Gunn, 2000). The failure of community mental health services to adequately meet the needs of those with mental illness, results in increased contact of these individuals with the CJS and prisons, and has been described as the criminalization of people with mental illness (Chaimovitz, 2012). As a result the CJS has taken on the role as a default provider of mental health care.

Policy in recent years has focused on the diversion of offenders with mental health problems into appropriate mental health services (Reed, 1992; Bradley, 2009). However the implementation of this policy has been inconsistent (McLeod et al., 2010), and joint training, specifically interagency training, has been proposed as a way of developing more responsive systems of early identification and diversion (Bradley, 2009; Hean et al 2011). 
The aim of this paper is to explore how participants at an interagency training day viewed interprofessional learning, and considers what interagency learning might offer in terms of promoting a more effective liaison and diversion response for offenders.

\section{The Diversion Agenda}

The aspiration behind managing the needs of individuals within the CJS who present with mental health problems, is through 'diversion' into mental health services. This initiative emerged following the Reed Report (1992), which recommended a national provision of diversion:

'There should be nationwide provision of properly resourced court assessment and diversion schemes and the further development of bail information schemes' (Reed Report, 1992; para 5.3.6).

As well as the benefits to the individual in terms of diversion into more appropriate mental health support, it has been suggested that the approach is cost affective, saving more than $£ 600,000$ per individual over the course of their lifetime (Renshaw, 2010). However the continued prevalence of mental illness in prisons demonstrates that diversion attempts are often unsuccessful, or fail to take place as there is no service operating in certain areas. The reasons for this are complicated and include poor implementation of the policy and under-funding (Sainsbury Centre for Mental Health, 2008). Another key issue involves the range of stakeholders and different cultural and organisational perspectives which underpin practice. Staff employed in the CJS and health and social care sectors need to understand how other agencies work, and how working together can improve offender outcomes (Hean, 2011). This includes better understanding of the range of services available to support early identification of mental health issues (Hean et al. 2009).

Despite recommendations for a diversion policy (Reed, 1992: Bradley, 2009), it has been claimed that the current system inhibits interagency working and accountability, resulting in offenders with mental health problems receiving inconsistent support throughout their court experience (McLeod et al., 2010). 
The boundaries between agencies and the different cultures of practice can lead to problems for interagency understanding and co-operation, whereas close working relationships between the police, courts, prisons, mental health services and other agencies, can improve responsiveness to the diversion agenda (McLeod et al. 2010).

On-going concern about the large number of people with mental health or learning disabilities in prison in England and Wales led to a governmental review led by Lord Bradley (Bradley, 2009). This reinforced the importance of diversion by recommending a National Diversion Programme to roll out liaison and diversion services for all police custody suites and courts by 2014. Diversion within this context can be understood as

“... a process of decision making, which results in mentally disordered offenders being diverted away from the criminal justice system to the health and social care sectors. Diversion may occur at any stage of the criminal justice process: before arrest, after proceedings have been initiated, in place of prosecution, or when a case is being considered by the courts." (Bradley, 2009 p. 16).

Within this system of liaison and diversion, Criminal Justice Mental Health Teams should be responsible for ensuring continuity in an individual's mental health care when they are in contact with the CJS. These services represent collaborations between the mental health and criminal justice systems to improve diversion of offenders into mental health services. This includes improving recognition and screening, mental health assessment, and signposting to health and social care services when appropriate, and the new national programme has supported the roll out of 20 pathfinder sites to implement the liaison and diversion schemes across England and Wales (Clapper, 2012). However, research into the effectiveness of these teams has indicated patchiness in provision, with many areas still having no provision (Pakes and Winstone, 2010).

\section{Mental illness and the Criminal Justice System}


People with mental health conditions and learning disabilities tend to experience greater difficulties in accessing justice than other groups, and are one of the most socially excluded groups within society as they experience greater discrimination, disadvantage and stigma (Mind, 2001). This is equally true for young people who offend or who are at risk of offending, who often have considerable mental health needs which may often go unmet. This may result in psychological, social, structural and cultural barriers to early identification of mental health needs and appropriate intervention (Walsh et al., 2011). Research suggests that understanding earlier life experiences of social exclusion are important in this population. For example, prisoners are 13 times more likely to have been in care as a child; 13 times more likely to have been unemployed; and 10 times more likely to have been a regular truant when at school (Social Exclusion Unit, 2002). Certain groups within the CJS may be at increased risk of experiencing mental health problems. Eight out of 10 women in prisons will suffer from diagnosable mental health problems (WHO, 2007), and women have been found to experience increased risk of self harm within the prison system (Short et al. 2009). Delays in identifying mental health problems for offenders means that they are often not diverted into more appropriate mental health provision and support.

Increased numbers of individuals within the CJS with mental health problems could be viewed as an unforeseen consequence of the policy of the closure of longstay hospitals (Arboleda-Florez and Holley, 1998). A move towards care in the community away from long stay institutions was a result of policies such as 'Better Services for the Mentally Ill (DHSS 1975), 'Care in the Community' (DHSS 1981) and 'Community Care with Special Reference to Mentally Ill and Mentally Handicapped People' (House of Commons' Social Security Committee 1985). These policies sought to provide more appropriate community based services to support individuals with mental health needs or learning disabilities. The motivation for these changes was a belief that care in the community would improve outcomes for those with mental health problems, and for many care provided within community settings has improved wellbeing and quality of life. However, research has suggested that significantly more people with serious mental health problems, such as 
schizophrenia, are convicted of almost all categories of criminal offences (Mullen et al. 2000), and this results in police officers, as well as other members of the CJS, coming into greater contact with offenders experiencing severe mental health problems (Cummins, 2006). This brings into sharp focus their underpinning knowledge of mental illness and the responses available within the diversion and liaison agenda. Within England a range of general legislation informs mental health practice, including the Mental Capacity Act (Department for Constitutional Affairs, 2007), the Mental Health Act 1983 (amended 2007) (Department of Health, 2007) and the Equality Act 2010 (HMSO, 2010).

\section{Joint training}

To support a robust system of diversion the Bradley Report (2009) made recommendations about the role of joint training in supporting the implementation of the diversion policy. The report suggested that

"Where appropriate, training should be undertaken jointly with other services to encourage shared understanding and partnership working. Development of training should take place in conjunction with local liaison and diversion services (Bradley, 2009, p. 111).

Joint training can develop a wider understanding of mental health for staff employed in CJS roles, including an increased awareness of how social issues and deprivation are linked to mental health and offending (James, 2010). The approach of CJS in the UK is overwhelmingly on punishment and risk reduction, and it has been suggested that this disadvantages further those who are already socially excluded (Duckett and Schinkel, 2008).

Joint training can encourage agencies to reflect upon their own role in supporting offenders with mental health problems, and how they can draw on the expertise of other agencies to work more effectively with them. Equally practitioners in health and social care settings may improve their response to offenders with mental health issues if they have a better understanding of the CJS. Other benefits include 
understanding of the constraints within which each service works and the appreciation of the working processes which facilitate enhanced communication between services. For example, since the mid-1990s, when the requirement for probation officers to obtain a social work qualification was rescinded in England and Wales, the distance between social work and probation practice has widened (National Offender Management Service (NOMS), 2005). Social workers in mental health settings may have little understanding of criminal justice and this has implications for the way they work with offenders with mental health needs. This is a global issue, and in the US it has been suggested that social work has neglected adults involved in the criminal justice system for nearly forty years (Pettus-Davis, 2012). Interagency training may enable social work agencies to re-frame their role in supporting the mental health needs of offenders who are diverted away from the CJS, as well as developing understanding of the role of other agencies involved in the diversion agenda.

Proposed changes to the way probation services are managed, through the involvement of private providers for 'low risk' offenders (Travis, 2013), raises the issue of whether these new agencies will be adequately trained in mental health awareness? If they lack the appropriate knowledge and skills it may lead to delays in the early identification of mental illness, resulting in problems within the CJS in terms of risk assessment and management. Further training is necessary for staff at all levels, to improve awareness of complex mental health needs and of measures to support early identification of such needs. However, there is evidence that working at this interface of the criminal justice system and mental health services is challenging (Hean et al., 2009), and increased opportunities for interagency training are required to facilitate understanding across professional groups (Hean et al., 2011).

To facilitate discussion about the benefits of joint training a workshop grounded in a 'crossing boundary' approach described by Engeström, (2001) was delivered in December 2011 to a sample of 52 professionals from a range of nonhealth professionals associated with the CJS (probation, police and courts) and professionals from the mental health system or health domain (learning disability, substance misuse and mental health services). This workshop focussed on enhancing interagency working between the different agencies. Participants were professionals working in two counties in the South of England and consisted of managers and 
practitioners in the following agencies: Appropriate adults; assertive outreach teams; court staff; crown prosecution service; community mental health teams; crisis and home treatment teams; court and custody diversion and liaison schemes; forensic medical examiners and police custody nurses; forensic mental health; the judiciary and magistrates; learning disability services; police; probation and substance misuse services ( $\mathrm{n}=52$ participants). The workshop was free of charge to promote attendance.

The following section considers the findings from focus groups which took place during the workshop to explore participant perceptions of the issues involved in interagency working and the content required to respond effectively to the liaison and diversion agenda set out by the Bradley Report (2009). A full report, of the wider findings of the workshop, is currently being developed.

\section{Focus Groups}

In the second half of the workshop, participants were divided into six focus groups (9-12 participants each), divided by agency to form a heterogeneous mix by profession in each group. Each group was facilitated by a single coordinator following a common interview schedule and set of prompts.

Participants were asked during these focus groups to reflect on two main themes:

- how to prepare the workforce to effectively respond to a liaison/diversion agenda;

- the constraints they worked under in terms of delivering training for professionals aiming to respond effectively to the diversion/liaison agenda.

Participants received information about data collection, confidentiality and anonymity in reporting. Written informed consent to record and report focus group discussions was collected before the session began.

Recordings were transcribed and a descriptive textual analysis conducted. Three of the research team engaged in the analysis. A process of familiarisation took 
place for each researcher via data immersion through reading and re-reading the transcripts. As the questions of the framework were on the content of training and the mode of delivery, this dual structure was imposed on the data. Once achieved, key concepts were identified from the data itself to construct a framework for communicating the essence of what the data highlighted. To promote the dependability of the qualitative analysis, the research team met to agree the categories and emerging themes.

\section{Findings}

The responses of participants were categorised into three general themes: the content of any potential training, what the delivery method might be for the content and lastly how commissioning might occur. This paper focuses specifically on the participant perspectives concerning the content required to support effective interagency training.

\section{Awareness of diversion and liaison agenda}

Participants identified knowledge and skills that they felt professionals required to respond to the diversion and liaison agenda. A key training need appears to be developing understanding of mental health or criminal justice systems and how these systems can work together effectively in order to support offenders with mental health needs.

\footnotetext{
'For me as a care co-ordinator I would have no knowledge of the criminal justice system and I think that's going to be key if you've got social workers or whoever in this court liaison service or nurses, having that criminal justice training I think would be very important definitely.'
}

A need identified by both criminal justice workers and mental health professionals concerned a lack of understanding of the liaison and diversion agenda. This was particularly important in identifying the positive impact of diversion and what it meant for working across systems. 
'[We] need to understand the purpose of it [the diversion agenda] in the first place and the issues behind the necessity for [it] so that you've got a group of staff working with...mental health problems...that understand what the issues are for those offenders in the first place.'

It is vital that all practitioners coming into contact with offenders with mental health needs are aware of both the nature of mental illness as well as the services available to support the diversion agenda.

\section{Awareness of roles, targets and legal responsibilities of other agencies}

There was an acknowledgement that different agencies had little understanding in three main areas: others' roles, others' targets and the legal responsibilities and policies of other agencies. All were seen as necessary to improve interagency working and ultimately to improve outcomes for offenders with mental health needs. Participants identified a need to understand the wider systems for providing offender mental health support and the roles of different agencies in this process.

'What has always struck me is how little different agencies understand each other's roles and therefore for us all in the system to be effective,... there needs to be some awareness, education even in terms of what that system actually is and who's involved and all of the services.'

'I would like to see officers at a training level equipped with a greater understanding of mental health problems and disorders. They are not experts, that's not their job, they're police officers, and I get that bit, but if they knew a little bit more, and vice versa, if we knew a little more about how the criminal justice system works and your expectations of us, I think the relationship would improve no end really. This agenda that we're talking about today might move on.' 
Understanding the wider systems of criminal justice and mental health also involves developing insights into the targets and priorities of other agencies.

Participants felt it was important to understand what other agencies had to achieve, what was imposed on them in terms of targets and how this influenced their decision- making in terms of diversion of offenders with mental health problems.

'I think what was said this morning about magistrates have got their targets, police have got their targets, every agency, especially as you said with funding being cut and cut you are more and more expected to deliver to your targets and if those conflict with partners'[targets] it's very difficult to work to the same agenda.'

'One of the issues that struck me was the differing priorities that differing agencies have....'

This raises the importance of practitioners understanding the wider organisational and policy requirements and constraints which structure practice across criminal justice and mental health settings. This includes understanding relevant legislation, policy and agency requirements.

\section{Facilitating cultural understanding}

Interagency training could help agencies understand the different organisational cultures which exist across the different worlds of criminal justice and mental health. It could support individuals and agencies to understand the different 'communities of practice' (Wenger 1998) which exist across stakeholder groups. Deeper understanding of different perspectives could help to facilitate a move to a shared culture of understanding concerning the liaison and diversion agenda. Participants identified this as a need to understand the 'culture' of other agencies.

'Actually if we could have a magic wand... if we could suddenly, not necessarily change your culture, but share an understanding of your culture... 
why your culture is this and this is your rules. That would facilitate working. But it's how would we do that considering the constraints.'

Understanding the cultural context of agencies also involves developing a deeper understanding of the statutory duties and legal framework they operate within. Many agencies, both in mental health, drugs and alcohol and criminal justice are underpinned by statutory duties and legal framework. Participants were keen to know more about these as they felt it influenced their work with other agencies. This was also articulated as a need to understand what pressures other agencies were under so that other agencies had realistic expectations of them.

'I think that knowledge of how the criminal justice systems works is critical especially for crisis teams, the P.A.C.E pressures, ${ }^{1}$ and the speed at which the criminal justice system must process people doesn't give time for crisis services... to respond in a way that they normally would.'

'Especially when those agencies are dealing with different local authorities, different primary care trusts who all have their own policies and protocols so you cross a border and no policy is the same as where you were and so it's getting a standard across the board which is very difficult and you're dealing with so many different agencies.'

A key aspect of facilitating cultural understanding was felt to be through enhancing communication skills. Interagency training was seen as a means of enhancing communication skills and communication channels between professionals from different agencies especially between senior managers across agencies, thereby optimising a cultural change towards interagency working.

'I think there's got to be a whole culture change..whereby...people at a strategic level start to look more across agencies. .. We probably see it as coal face workers what we need to do, but unless it's driven from a much higher level...'

\footnotetext{
${ }^{1}$ Police and Criminal Evidence Act 1984.
} 
'I think things can be improved if the managers liaise more closely.

'I think the way you do that initially would be to afford everybody with a clear idea about what we all do and how we will communicate with each other.'

\section{Challenging prejudice}

Facilitating improved communication and cultural understanding of the context of mental and health and criminal justice provision was also felt to be important in terms of producing attitudinal change. Attitudinal change occurs when deep seated prejudices are challenged and discrimination attached to both the labels and stigma attached to 'mental illness' and 'offending' are re-examined. It supports individuals and agencies to understand the wider impact of social exclusion and discrimination on offenders with mental health problems, and how this may militate against early identification and appropriate and timely diversion.

'I think frankly sometimes there's some prejudice inside mental health services to people [who] offend.'

'I would actually say perhaps more general training for magistrates about stigma and discrimination...'

This raises the importance of interagency training as providing a 'space' for practitioners to explore assumptions and prejudice attached to mental health and offending which may be perpetuated by particular agency cultures. Dialogue and communication may enable individuals and agencies to reflect upon the importance of challenging stigma and prejudice and viewing the wider impact of social exclusion related to this field.

\section{Discussion}

The policy of liaison and diversion is central to UK Government policy, with the aim of a national diversion programme rolling out liaison and diversion services 
for all police custody suites and courts across England and Wales by 2014. Due to the current patchy implementation of this policy (McLeod et al., 2010), it is important to consider factors which may promote the effective development of such schemes.

The issues emerging from the focus group discussions confirm the importance of developing interagency awareness and understanding to support the implementation of the diversion agenda. The views expressed by participants within the focus groups support the findings of earlier work which highlighted that increased opportunities for interagency training are required to facilitate understanding across professional groups (Hean et al., 2011).

During the focus groups participants stressed the importance of interagency training to provide opportunities for deeper understanding of the different cultures and ethos that exist across the fields of mental health and the CJS. This is important in supporting understanding of how 'communities of practice' (Wenger, 1998) may develop within and across organisational boundaries. During the workshop, participants were able to explore how organisational boundaries 'influence the ability ...to exert an influence over other organisations or groups' (Hernes 2004, p. 9), and in turn how this impacts upon early identification of mental health issues and the diversion agenda.

Coming together with a wide range of practitioners from across different agencies allows individuals to understand how different values and philosophies inform practice across different organisational contexts. This highlights how shared understanding can improve responsiveness to early detection and identification of mental health issues. Such activity helps to develop new cultures of understanding across different stakeholder boundaries.

Interagency training provides an opportunity for participants to develop awareness of roles, targets and legal responsibilities of other agencies. Although all agencies are ultimately concerned with the welfare of the offender, there are competing agendas which permeate the roles and tasks which agencies are required to carry out. A focus on public safety and risk reduction is an essential role for criminal justice workers, whereas health and social care professionals are more concerned with the welfare of the person with mental health needs and a commitment to anti- 
discriminatory and anti-oppressive practice (Fenton, 2012). Understanding the targets that certain groups need to meet helps to facilitate wider understanding of the system as a whole.

An interesting issue to emerge was the perspective that interagency learning can support practitioners and agencies to challenge prejudice; for example, the suggestions from participants that magistrates and the police need to develop a deeper understanding of mental health within a wider social context of exclusion and discrimination. Interagency training can develop understanding of how offenders with mental health problems may be disadvantaged by the systems put in place to meet their needs. Individuals or groups can be excluded by 'social boundaries' from other groups and from society as a whole (Madanipour et al. 1998), and those who offend and have mental health problems may be doubly disadvantaged and excluded. This is particularly important in light of the suggestion that prisoners are probably the most socially excluded group in our society (Rutherford et al. 2008).

Overall, the participants held positive attitudes towards their experience of interagency training and its benefits. Of particular importance is the way in which face-to-face contact and discussion of case studies enabled participants to develop knowledge and understanding of the diversion and liaison agenda. Other key content identified concerned the roles and tasks of different agencies, cultural contexts of practice, and the opportunity to challenge prejudice.

\section{Limitations}

This paper reports on a small scale interagency learning event, and there is scope to explore the transferability of these perspectives to the wider national population of professionals in the mental health and CJS.

\section{Conclusion}

Liaison and diversion is at the heart of government policy to support offenders with mental health needs, yet the implementation of this scheme has been patchy to date. This paper suggests that interagency training can support practitioners across the 
CJS and health and social care sectors to become more responsive to the diversion policy and work more effectively together.

Interagency training offers an approach to optimising the way these agencies collaborate with one another, and facilitates understanding of the roles and tasks of all involved in the support of offenders with mental health needs. Focus group feedback suggests that professionals from both the mental health and CJS are keen to the understand the culture of other agencies. There appears to be a need for professionals in both the MHS and CJS to develop an awareness of the roles, targets and legal responsibilities of other agencies. The challenge is how government and agencies invest in the development and implementation of this training which appears central to embedding the diversion agenda within national practice.

\section{ACKNOWLEDGEMENTS}

We are grateful to all the participants from the mental health services and CJS who attended the interagency workshop upon which this paper is developed.

\section{REFERENCES}

Aboleda-Florez, J. \& Holley, H. L. (1998) 'Criminalization of the mentally ill: Part II, Initial detention. Canadian Journal of Psychiatry, (33): 87-95.

Bradley, Lord. (2009). 'The Bradley Report.' London: Department of Health.

Retrieved from http://www.dh.gov.uk/prod_consum_dh/groups/dh_digitalassets/documents/digitalass et/dh_098698.pdf [accessed 01/02/2013]

Chaimovitz, G. (2012) The Criminalization of People With Mental Illness, The Canadian Journal of Psychiatry, 57(2): 1-6. 
Clapper, A. (2012) Mapping Liaison and Diversion Services for Hampshire and the Isle of Wight A Report for NHS South of England. Lymington: Andican Consultancy.

Cummins, I. (2006) 'A Path Not Taken? Mentally Disordered Offenders and the Criminal Justice System', Journal of Social Welfare \& Family Law. 28, (3-4): 267281.

Department of Constitutional Affairs (2007) 'Mental Capacity Act 2005

Code of Practice', London: HMSO.

Department of Health and Social Security (1975) 'Better Services for the Mentally Ill'. London: HMSO.

Department of Health and Social Security (1981) 'Care in the Community', London: HMSO.

Department of Health. (2007a) Improving Health, Supporting Justice.

London: Stationary Office, Department of Health.

Department of Health (2007b) Mental Health Act 2007. London: HMSO.

Department for Work and Pensions (2005) Disability Discrimination Act, London: HMSO.

Duckett, P.and Schinkel, M. (2008) 'Community Psychology and Injustice in the Criminal Justice System', Journal of Community \& Applied Social Psychology, 18: $518-526$.

Engestrom Y. (2001) 'Expansive learning at work: towards an activity theoretical reconceptualisation', Journal of Education and Work, 14: 133-156.

Equality Act (2010) London: The National Archives. [Accessed16/08/2013]. 
Fenton, J. (2012) 'Bringing Together Messages from the Literature on

Criminal Justice Social Work and 'Disjuncture': The Importance of 'Helping',

British Journal of Social Work, 42: 941-956.

Gunn, J. (2000) 'Future directions for treatment in forensic psychiatry', British Journal of Psychiatry', 176: 332-338.

Harcourt, B. (2006) From the Asylum to the Prison: Rethinking the Incarceration Revolution, Texas Law Review, 84(7): 1751-1786.

Hean, S., J. Warr, \& Staddon, S. (2009) Challenges at the interface of working between mental health services and criminal justice system. Medicine, Science and the Law, 49(3), 170-178.

Hean, S., Heaslip, V., Warr, J., and Staddon, S. (2011) Exploring the potential for joint training between legal professionals in the criminal justice system and health and social care professionals in the mental-health services, Journal of Interprofessional Care, 25: 196-202.

Hernes, T. 2004 'Studying composite boundaries: A framework of analysis', Human Relations, 57 (1): 9-29.

House of Commons Social Services Committee (1985) Community Care With Special Reference to Mentally Ill and Mentally Handicapped People. London: HMSO.

James, D.V. (2010) 'Diversion of mentally disordered people from the criminal justice system in England and Wales: An overview', International Journal of Law and Psychiatry, 33: 241-248.

McLeod, R., Philpin, C., Sweeting, A., Joyce, L. and Evans, R. (2010) 'Court experience of adults with mental health conditions, learning disabilities and limited mental capacity Report 1: Overview and recommendations', Ministry of Justice Research Series 8/10, Ministry of Justice: Crown Copyright. 
Madanipour, A., Cars, G. and Allen, J. (Eds.) 1998. Social Exclusion in European Cities: Processes, Experiences and Responses. London: Jessica Kingsley.

Mind (2001) Silenced Witness. London: MIND.

Ministry of Justice (2007) Statistical bulletin: Statistics of Mentally Disordered Offenders England and Wales 2006, London, Ministry of Justice.

Mullen, P., Burgess, P., Wallace, C., Palmer, S. and Rusctiena, D. (2000) 'Community care and criminal offending in schizophrenia', The Lancet, 355 : 614617.

National Offender Management Service (NOMS) (2005) The National Probation Service for England and Wales, published online at http://webarchive.nationalarchives.gov.uk/

20101123002005/http://probation.homeoffice.gov.uk/files/pdf/The\%20National\%20P robation\%20Service\%20for\%20England\%20and\%20Wales\%20Leaflet.pdf. [accessed 01/02/2013]

Pakes, F. and Winstone, J. (2010) 'A site visit survey of 101 mental health liaison and diversion schemes in England', The Journal of Forensic Psychiatry \& Psychology, 21 (6): $873-886$.

Pettus-Davis (2012) 'Reverse Social work's Neglect of Adults in the Criminal Justice System: The Intersection and an Agenda', Social Work Research, 36 (1): 3-8.

Reed, J. (1992) Review of health and social services for mentally disordered offenders and those requiring similar services. Department of Health/Home Office. London: HMSO.

Renshaw, J. (2010) Waiting in the Wings, London: Laing and Buisson. 
Rutherford,M., Samele, C. and Duggan, S. (2008) 'Building momentum: how the Sainsbury Centre for Mental Health's Prisons and Criminal Justice Programme is contributing to the pressure for change', Criminal Behaviour and Mental Health, 18: 261-267.

Sainsbury Centre for Mental Health (2008) The Police and Mental Health, Briefing Paper 36. London: Sainsbury Centre for Mental Health.

Short, V., Cooper, J., Shaw, J., , Kenning, C., Abel, K., and Chew-Graham, C. (2009) Custody vs care: attitudes of prison staff to self-harm in women prisoners-a qualitative study, The Journal of Forensic Psychiatry \& Psychology, 20, (3) , 408426.

Singleton, N., Meltzer, H., Gatward, R., Coid, J., \& Deasy, D. (1998)

Psychiatric morbidity among prisoners in England and Wales: the report of a survey carried out in 1997 by Social Survey Division of the Office of National Statistics on behalf of the Department of Health, London: Stationary Office.

Social Exclusion Unit. (2002) Reducing reoffending by ex-prisoners. London: HMSO.

Social Exclusion Unit (2004) Mental Health and Social Exclusion, Office of the Deputy Prime Minister., London: HMSO.

The Equality Act (2010), London: HMSO.

Travis, A. (2013) Probation service 'revolution' means wholesale privatisation, The Guardian. Available from http:www.guardian.co.uk/society/2013/jan/09/probation-service-private-firm grayling \{ Accessed 04/02/13).

Van Marle, H,J, C. (2007) Mental Health care in prison: how to manage our care, International Journal of Prisoner Health, 3:115-123. 
Wacquant, L. (2010) Crafting the Neoliberal State: Workfare, Prisonfare, and Social Insecurity, Sociological Forum, 5(2): 197-220.

Walsh, J., Scaife, V.,Notley, C., Dodsworth,J. and Schofield, G. (2011) 'Perception of need and barriers to access: the mental health needs of young people attending a Youth Offending Team in the UK', Health and Social Care in the Community, 19(4): $420-428$.

Wenger, E. (1998) Communities of Practice: Learning, Meaning and Identity, Cambridge: University Press

World Health Organisation (2007) Fact sheet: mental health and prisons, Copenhagen: WHO Regional Office for Europe. 